\title{
Partial cDNA Sequence Analysis of Myosin Va from Rainbow Trout (On- corhynchus mykiss) and Its Relationship to Myosin V Isoforms from Other Vertebrates
}

\author{
Kátia Gisele Oliveira Rancura ${ }^{1}$, Cesar Martins ${ }^{1}{ }^{*}$, Michelli Rivero Montaño ${ }^{1}$, Robson Francisco Car- \\ valho ${ }^{1}$, Alexandre Azevedo \\ ${ }^{I}$ Departamento de Morfologia, Instituto de Biociências, Universidade Estadual Paulista, Botucatu, São Paulo, Brazil; ${ }^{2}$ \\ Núcleo de Pesquisas Ecológicas e Sócio Ambientais de Macaé, Centro de Ciências da Saúde, Universidade Federal do \\ Rio de Janeiro, Macaé, Rio de Janeiro, Brazil
}

\begin{abstract}
Partial cDNA sequences of myosin V from rainbow trout Oncorhynchus mykiss were analyzed and showed high similarity to MVa from other vertebrates. Phylogenetic analysis has shown that events resulting in the formation of paralogous copies of myosin $\mathrm{Va}, \mathrm{Vb}$, and $\mathrm{Vc}$ occurred before the divergence of vertebrates into different classes. Expression analysis of myosin $\mathrm{Va}, \mathrm{Vb}$, and $\mathrm{Vc}$ in different $O$. mykiss tissues revealed $\mathrm{MVa}$ exclusively expressed in hypophysis and brain whereas $\mathrm{Vb}$ and $\mathrm{Vc}$ were expressed in practically all tissues analyzed. The nucleotide sequence for myosin $\mathrm{V}$ was explored in a fish species for the first time and these results represent an important start in understanding the organization, evolution, and expression of myosins in early vertebrates. The data presented here represent contributions to the knowledge of rainbow trout genome. A better understanding of this economically important species could assist in development of improved strains of this fish for aquaculture.
\end{abstract}

Key Words: Nucleotide sequence, Amino acid sequence, Evolution, Tissue expression, Fish.

\section{INTRODUCTION}

Myosins make up a large superfamily of molecular motors that appeared early in eukaryotic evolution $[1,2)$. They are mechanoenzymes that bind to actin and hydrolyze ATP to produce mechanical force; their members participate in activities as diverse as cytokinesis, muscle contraction, and organelle motility [3].

Myosins are typically composed of three functional subdomains: (1) the motor domain which interacts with actin and binds ATP, (2) the neck domain which binds light chains or calmodulin, and (3) the tail domain involved in proteinprotein interactions. Although most myosins have the same general structure, the nonmotor regions vary enormously. This structural diversity reflects functional diversity [4]. Myosin heavy chains have been categorized into 18 structurally distinct protein classes, mostly based on comparisons and phylogenetic analysis of the conserved motor domain. However, current studies of the myosin gene family have revealed 37 myosin types with different protein domain combinations and scattered taxonomic distribution [5].

Class V myosin is one of the most ancient and extensively studied group of the myosin superfamily and the wide range of species in which it has been identified suggests that myosin $\mathrm{V}$ is a fundamental component of organelle transport in all higher eukaryotes [6]. It was initially characterized as an unusual calmodulin binding protein from the brain with a number of myosin-like biochemical properties [7,8,9]. Subsequently, myosin $\mathrm{V}$ heavy chain genes were cloned from

*Address correspondence to this author at the Cesar Martins, Departamento de Morfologia, Instituto de Biociências, UNESP - Universidade Estadual Paulista, 18618-000, Botucatu, SP, Brazil; Tel/Fax: ++55(14)38116264, E-mail: cmartins@ibb.unesp.br mouse, yeast, and chicken, thus defining the fifth class of actin-based motors $[10,11,12,13,14]$. Vertebrate class V myosins have three members, $\mathrm{Va}, \mathrm{Vb}$, and $\mathrm{Vc}$. Myosin Va has undergone the most investigation and is expressed at high levels essentially in the brain and melanocytes. The second member, myosin $\mathrm{Vb}$ has been cloned from rat [15], but has received relatively little attention. The third member, myosin $\mathrm{Vc}$ was discovery and cloned recently and is relatively abundant in many secretory and glandular tissues, where it is predominantly expressed in epithelial cells [16].

The presence of the three class $\mathrm{V}$ members of myosins among vertebrates leads to many questions on their relative roles within the cell related to expression and tissue distribution. Considering that little attention has been direct to myosin genes in ancient vertebrates, in this study we investigated myosin V-encoding cDNAs in Oncorhynchus mykiss rainbow trout aiming to contribute to the basic knowledge on its functions and evolutionary behavior in early vertebrates. Raibow trout originate from western North America and were widely introduced in several coutries of the world, including Brazil [17]. Although genetic linkage maps and physical chromosome maps have been constructed for rainbow trout [18], genome information of this species are still needed to assist in development of improved strains of this fish for aquaculture.

\section{MATERIALS AND METHODOLOGY}

Adult rainbow trout (O. mykiss) specimens were obtained from Núcleo Experimental de Salmonicultura de Campos do Jordão (São Paulo, Brazil). This trout stock was originated from imported animals from Mount Shasta, California, USA. The animals were sacrificed with an overdose of benzocaine followed by spinal transection (Protocol 01204 Ethical Committee on Animal Experimentation - Instituto de 
Table 1. Primer Sequences and Annealing Region on Myosin V Domains

\begin{tabular}{|c|c|}
\hline Functional Domains & Primer Sets \\
\hline \hline Head & BMV-F (5'-CTGGTGGGTGAGAATGACCT-3') \\
& BMV-R1 (5'-CTTGATGCAGCGCACATAGT-3') \\
\hline Neck & MyRC1 (5'-AAGACTGTTGGCTGCCAGTT-3') \\
& BMV-R (5'-GCAGCTGCATGATCTTGTTT-3') \\
\hline Tail Va, Vb, and Vc & Tail-Va-F1 (5'-TAGAAGTGGGGCAGATGGAG-3') \\
& Tail-Va-R1 (5'-ACTGGAGGTTCGCTTTCTGA-3') \\
& Tail-Vb-F1 (5'-TGAGAGTATCCAGGGGCTGT-3') \\
& Tail-Vb-R1 (5'-AGCATCCTCCTGGGTCTTCT-3') \\
& Tail-Vc-F1 (5'-CCAAGCAACTTTTGGAGAGC-3') \\
& Tail-Vc-R1 (5'-CTGCTTGCTTCTTTGGGAAC-3') \\
\hline
\end{tabular}

Biociências, UNESP, Botucatu, Brazil) and hypophyses, brains, aortas, hearts, gills, muscles, stomachs, livers, kidneys, intestines, and spleens were dissected and frozen in liquid nitrogen for subsequent RNA extraction.

Total RNA samples were obtained using TRIzol reagent (Invitrogen) as per manufacturer's instructions. RNA was quantified by spectrophotometry (Biophotometer Eppendorf) at $260 \mathrm{~nm}$ and $280 \mathrm{~nm}$, using the RNA correction factor. Firststrand cDNA synthesis reaction was performed with "SuperScriptTM First-Strand Synthesis System for RT-PCR" commercial kit (Invitrogen Life Technologies) using random hexamer primers. The cDNA amplification was performed using BM5-F and BM5-R1 primer sets for the head domain, and MYCR1 and BMV-R for the neck domain of myosin $\mathrm{Va}$, and Tail-Va-F1 and Tail-Va-R1, Tail-Vb-F1 and TailVb-R1 and Tail-Vc-F1 and Tail-Vc-R1 for the tail domains of myosin $\mathrm{Va}, \mathrm{Vb}$, and $\mathrm{Vc}$, respectively (Table 1). The primer sets employed were designed from published chicken myosin Va cDNA sequence (GenBank accession number NM205300), genomic nucleotidic sequence of zebrafish (GenBank accession number BX088562), and rat myosin V cDNA sequences (GenBank accession numbers AB035736, U60416, and XM236411).

RT-PCR products were visualized by $1 \%$ agarose gel electrophoresis and cloned in plasmid pCR2.1 (kit TA Cloning - Invitrogen) and pGEM-T (Promega) and used to transform E. coli DH5 $\alpha$ strain competent cells (Invitrogen Life Technologies). The recombinant plasmids containing genes of interest were purified with the "Wizard Plus Minipreps DNA Purification System" kit (Promega) and submitted to nucleotide sequencing on an ABI 377 Automated DNA Sequencer (Applied Biosystems).

Table 2. Species, Myosin Isoforms and Domains Analyzed, and Accession Numbers of Amino Acid Sequences from Several Organisms Obtained from NCBI Databases

\begin{tabular}{|c|c|c|}
\hline Species & Myosin Isoform and Domain & Accession Numbers \\
\hline Bos taurus & Vc head and neck & XP_611694 \\
\hline Bos taurus & Va tail & XM_615219 \\
\hline Bos taurus & Vc tail & XM_611694 \\
\hline Canis familiaris & Vc head and neck & XP_544680 \\
\hline Canis familiaris & Va tail & XM_535487 \\
\hline Canis familiaris & $\mathrm{Vb}$ tail & XM_537345 \\
\hline Canis familiaris & Vc tail & XM_544680 \\
\hline Danio rerio & Vc head and neck & XP_691143 \\
\hline Gallus gallus & Va head and neck & CAA77782 \\
\hline Homo sapiens & Va head and neck & NP_000250 \\
\hline Homo sapiens & $\mathrm{Vb}$ head and neck & XP_944193 \\
\hline Homo sapiens & Vc head and neck & EAW77446 \\
\hline
\end{tabular}


Table 2. contd.....

\begin{tabular}{|c|c|c|}
\hline Species & Myosin Isoform and Domain & Accession Numbers \\
\hline Homo sapiens & $\mathrm{Vb}$ tail & XM_939100 \\
\hline Homo sapiens & Vc tail & NM_018728 \\
\hline Macaca mulatta & Va head and neck & XP_001084476 \\
\hline Macaca mulatta & $\mathrm{Vb}$ tail & XM_001090668 \\
\hline Mus musculus & Va head and neck & NP_034994 \\
\hline Mus musculus & $\mathrm{Vb}$ head and neck & NP_963894 \\
\hline Mus musculus & Vc tail & XM_983747 \\
\hline Pan troglodytes & Vc head and neck & XP_510411 \\
\hline Pan troglodytes & Va tail & XM_001170332 \\
\hline Pan troglodytes & $\mathrm{Vb}$ tail & XR_024243 \\
\hline Pan troglodytes & Vc tail & XM_510411 \\
\hline Rattus norvegicus & Va head and neck & NP_071514 \\
\hline Rattus norvegicus & $\mathrm{Vb}$ head and neck & NP_058779 \\
\hline Rattus norvegicus & Vc head and neck & XP_236411 \\
\hline Tetraodon nigroviridis & Va head and neck & CAG00830 \\
\hline Tetraodon nigroviridis & $\mathrm{Vb}$ head and neck & CAG01035 \\
\hline Xenopus laevis & Va head and neck & AAH45050 \\
\hline Strongylocentrotus purpuratus & $\mathrm{V}$ head and neck & NP_999655 \\
\hline Caenorhabditis elegans & V head and neck & NP_505433 \\
\hline Drosophila melanogaster & V head and neck & AAC99496 \\
\hline
\end{tabular}

The sequences from myosin $\mathrm{V}$ head and neck were processed by CAP3 software, available at http://pbil.univlyon1.fr/cap3.php, to obtain a unique contiguous sequence for this region. It was not possible to connect sequences from the myosin $\mathrm{V}$ tail portion with the head-neck segment. The resulting cDNA and predicted aa sequences were subjected to Blast $/ \mathrm{n}$ and $B l a s t / x$ searches, respectively [19] at the NCBI website http://www.ncbi.nlm.nih.gov/blast. A further search for similarity to myosin $\mathrm{V}$ was performed against sequences contained in NCBI databse. Selected and representative sequences from different vertebrates were aligned using ClustalX [20] and molecular evolutionary analyses were conducted with MEGA version 3.1 [21], using the rainbow trout predicted aa sequences from the head and neck myosin domains and NCBI database myosin $\mathrm{V}$ aa sequences from other organisms (Table 2).

\section{RESULTS AND DISCUSSION}

RT-PCR amplification of $O$. mykiss myosin V from total RNA extracted from brain and other tissues allowed the amplification of a $2545 \mathrm{bp}$ band corresponding to MVa head and neck domains, and bands of 650bp for MVa, $400 \mathrm{bp}$ for $\mathrm{MVb}$ and 500bp for MVc from the tail domains. The RT-PCR DNA fragments were cloned and sequenced. Nucleotide sequence analysis through NCBI database searches by Blast/n indicated that rainbow trout isolated cDNA nucleotide sequences were highly similar to MV sequences of other vertebrates. 


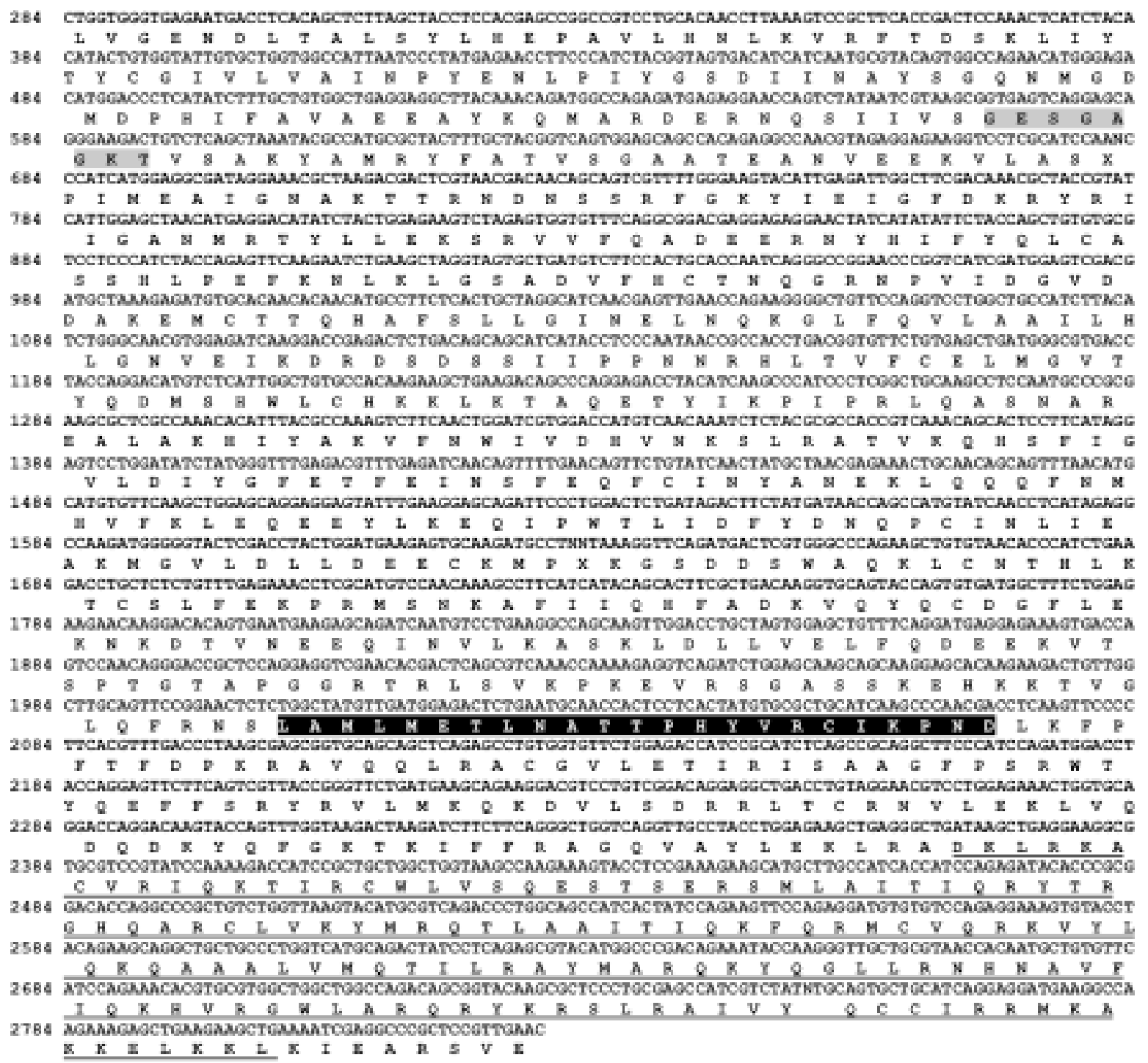

Fig. (1). Nucleotide and amino acid deduced sequences from trout brain myosin Va head and neck domains. The ATP-binding site is enhanced in gray and the actin-binding site in black. The underlined amino acid sequence denotes the myosin neck domain containing six IQmotifs. The nucleotide sequence is deposited in NCBI under accession number EF592540.

Brain MVa head and neck domain nucleotide sequences were obtained for four clones, edited with CAP3 software and manually, and resulted in fragments with 2545bp (Fig. 1). The deduced $O$. mykiss MVa head and neck aa sequence corresponds to aa residues 68 to 935 with high similarity to MVa from other vertebrates. Genetic distance analysis between the four cloned fragments of brain MVa head and neck domains revealed variant forms of MVa. The four isolated fragments were highly similar to each other (mean genetic distance of 0.0105). The variant transcripts detected could be related to polymerase errors during PCR or even sequence reading errors. On the other hand, variant transcripts related to alternative MVa splicing were detected in vertebrate tissues and were related to specific cellular process roles $[22,23,24,25]$.

All myosins share a core of conserved residues in their motor domains, many of which are known to participate in actin binding [26]. The ATP-binding site for MVa was highly conserved and no variation in nucleotide or aa sequences was observed in several non-related vertebrates.
This is probably due to the fundamental role of this region in energy conversion for the mechanical force required by the head domain for protein movement [6]. The actin-binding site was also well conserved for vertebrates with only two amino acid substitutions in residues 644 and $645(\mathrm{~A} / \mathrm{H}$ or $\mathrm{Q}$ and $\mathrm{M} / \mathrm{L}$ ). In the first substitution, a hydrophobic amino acid (alanine) was substituted by histidine or glutamine, which are both more water soluble, in the sequences of several organisms [27]. The second alteration occurs between aa with same properties, which should not cause damage in the protein structure because both are hydrophobic and important in stabilizing the protein structure by promoting hydrophobic interactions in its core [27]. Analyzed sequences showed the neck domain to be a more variable region, with several aa residue substitutions. However, most variable residues are neutral or basic. Even with such a variation, six imperfect aa repetitions can be recognized; these are called IQ-motifs $[28,29]$, a feature of class $\mathrm{V}$ myosins, represented by the consensus sequence IQXXXRGXXXR which are calmodulin and other light chain binding sites [31]. Comparative analy- 


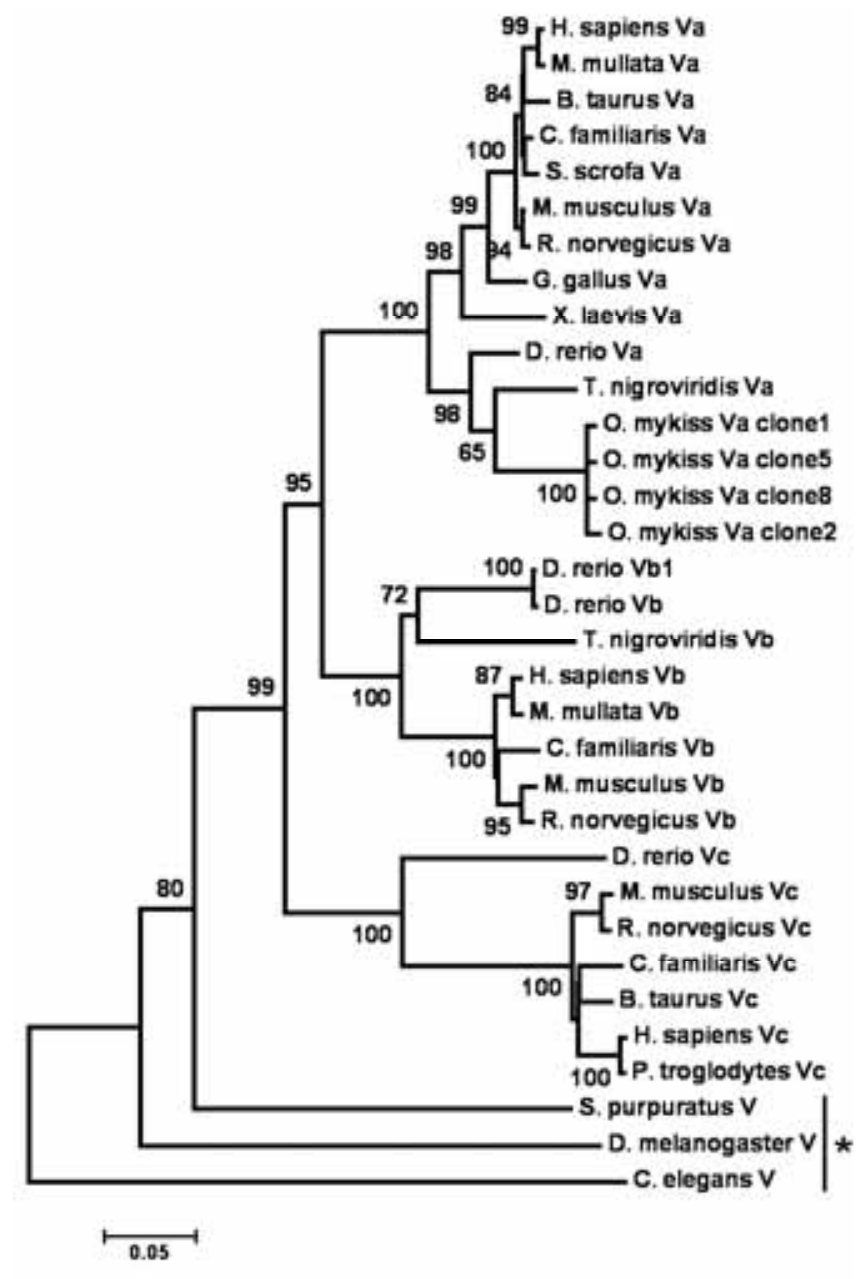

Fig. (2). Phylogenetic tree obtained from neighbor-joining analysis of amino acid sequences from rainbow trout brain and other vertebrate myosin Va head and neck domains. Sequences of nematodes, arthropods, and echinoderms (asterisk) were included as outgroups. Branch lengths are proportional to evolutionary distance (scale bar). Bootstrap resampling (2,000 trials) was used to judge the node robustness. Species NCBI accession numbers are described in Material and Methods.

sis of the myosin $\mathrm{V}$ head and neck domains amino acid frequency in several organisms and rainbow trout myosin $\mathrm{Va}$ showed a high frequency of neutral and basic amino acids such as Leucine and Lysine.

Genetic distance analyzes between myosin V from several vertebrates showed a mean genetic distance of 0.118 for MVa, 0.187 for myosin Vb, and 0.122 for myosin Vc. Comparative analysis of myosin $\mathrm{V}$ showed that rainbow trout MVa had a mean genetic distance of 0.118 in relation to $\mathrm{MVa}$ from other vertebrates, 0.278 to myosin $\mathrm{Vb}$, and 0.299 to myosin Vc. Rainbow trout MVa is closely related to the MVa and quite distant from MVb and MVc of several vertebrates. Trout MVa had lower genetic distance values than other fish species compared to other vertebrates. Such data can also be seen in phylogenetic reconstruction based on aa sequences from several vertebrates including rainbow trout (Fig. 2). Distant non-related species such as nematodes, arthropods, and echinoderms were included in the analysis as out-groups. The degree of similarity between sequences from different animal groups follows their phylogenetic distance. The event that resulted in the formation of paralogous $\mathrm{Va}$, $\mathrm{Vb}$, and $\mathrm{Vc}$ myosin copies occurred in a common ancestor before vertebrates diverged into different classes. This evolutionary event generated two copies of myosin V which diverged, one originated $\mathrm{MVc}$ in several vertebrates and the other generated the $\mathrm{Va}$ and $\mathrm{Vb}$ isoforms which were distributed in different vertebrate classes. It is interesting to note that, even within a specific vertebrate clade, all the myosin V members underwent evolutionary events which lead to divergences in a specific myosin class between different groups. All these evolutionary gene duplication and divergence events are supported in phylogenetic analyses by high bootstrap values (Fig. 2).

Tail domain nucleotide sequences for MVa, MVb, and MVc were submitted to searches by similarity in GenBank databases using the Blast/N program. Results showed a high similarity mainly for MVa sequences, with lesser values for MVb and MVc (Table 3). MVa tail was RT-PCR amplified in hypophysis and a very discreet band was also seen in brain (Fig. 3a); this agrees with literature which indicates that isoform is mainly expressed in nervous tissue [16,32]. Immunochemistry analysis suggest that $\mathrm{MVa}$ is involved in cell process such neurotransmission [33,34,35], growth cones motility in growing neurons [36] and translocation of cell organelles [37,38]. Mutations in the MVa cause the dilute pheno-

Table 3. Partial cDNA Nucleotide Sequence Similarity Levels (\%) for Myosin Va, Vb and Vc Between Rainbow Trout Tail Domain and Several Organisms Obtained from NCBI

\begin{tabular}{|c|c|c|c|c|c|}
\hline & O. mykiss Va & & O. mykiss Vb & & O. mykiss Vc \\
\hline M. musculus Va & 86 & M. musculus $\mathrm{Vb}$ & 54 & M. musculus $\mathrm{Vc}$ & 79 \\
\hline H. sapiens Va & 81 & H. sapiens $\mathrm{Vb}$ & 50 & H. sapiens $\mathrm{Vc}$ & 68 \\
\hline M. mulatta Va & 81 & M. mulatta $\mathrm{Vb}$ & 51 & M. mulatta $\mathrm{Vc}$ & -- \\
\hline S. scrofa Va & 81 & S. scrofa $\mathrm{Vb}$ & -- & S. scrofa $\mathrm{Vc}$ & -- \\
\hline C. familiaris Va & 80 & C. familiaris $\mathrm{Vb}$ & 50 & C. familiaris $\mathrm{Vc}$ & 68 \\
\hline
\end{tabular}




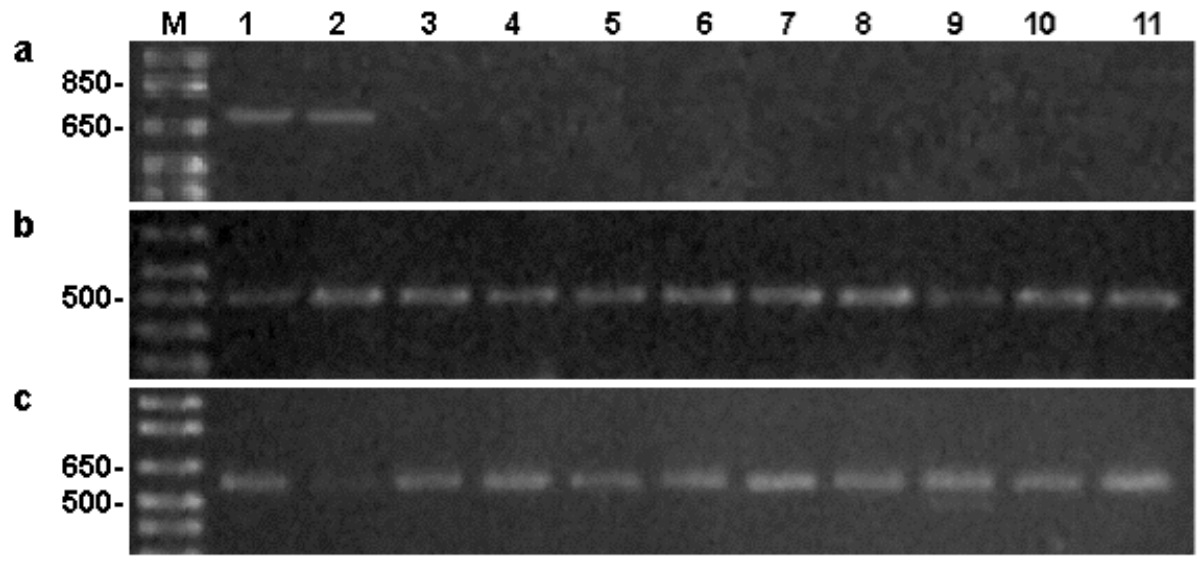

Fig. (3). 1\% agarose gel demonstrating MVa (a) MVb (b), and MVc (c) expression in different rainbow trout tissues. Legend: 1, hypophysis; 2, brain; 3, aorta; 4, heart; 5, gill; 6, muscle; 7, stomach; 8, liver; 9, kidney; 10, intestine; 11, spleen; and M, molecular weight marker in bp.

type in mice, which is associated to several neurological disorders that lead to death up to three weeks after birth [10]. The dilute fenotype causes de Griscelli's syndrome in humans that is characterized by neurological damages [39]. $\mathrm{MVb}$ and MVc isoforms were found in practically all tissues analyzed (Fig. 3b and 3c), with Vc more widely distributed and with a higher expression level in epithelial tissues. MVc is an actin-based motor protein involved in membrane trafficking of many physiologically crucial tissues of the human body [16]. Although the three MV classes are involved with membrane trafficking, several evidences have showed that the different myosin $\mathrm{V}$ isoforms are associated with a specific set of membrane trafficking events [16].

\section{CONCLUSIONS}

Comparative analysis of MVa between different vertebrate species clearly demonstrated that the evolution of myosins has accompanied the divergence of the main vertebrate groups. These data support the idea that conserved MV genes phylogeny is accompanied by conserved cellular functions. This is the first study to reveal the cDNA nucleotide sequence and expression of MV isoforms in rainbow trout. The data presented here represent new contributions to the knowledge of the genome of rainbow trout. A better understanding of this economically important species could assist in development of improved strains of this fish for aquaculture.

\section{ACKNOWLEDGEMENTS}

This work was supported by grants from FAPESP (Fundação de Amparo à Pesquisa do Estado de São Paulo), CNPq (Conselho Nacional de Desenvolvimento Científico e Tecnológico), and CAPES (Coordenação de Aperfeiçoamento de Pessoal de Nível Superior).

\section{ABBREVIATIONS}

$\begin{array}{lll}\text { aa } & = & \text { amino acid } \\ \text { ATP } & = & \text { Adenosine triphosphate } \\ \text { Blast } / \mathrm{N} & =\begin{array}{l}\text { Basic local alignment search tool for nu- } \\ \text { cleotide databases }\end{array} \\ \text { Blast } / \mathrm{X}= & \begin{array}{l}\text { Basic local alignment search tool for pro- } \\ \text { tein databases }\end{array}\end{array}$

$\begin{array}{lll}\mathrm{bp} & =\text { base pair } \\ \mathrm{CAP3} & =\text { Contig Assembling Program } \\ \text { DDBJ } & \text { DNA Data Bank of Japan } \\ \text { EMBL } & \text { European Molecular Biology Laboratory } \\ \text { GB } & \text { GenBank Data Base } \\ \text { IQ-motif } & \text { consensus sequence IQXXXRGXXXR that } \\ \text { bEGA } & \text { Molecular Evolutionary Genetics Analysis } \\ \text { MVa } & \text { Myosin Va } \\ \text { NCBI } & \text { National Center for Biotechnology Infor- } \\ \text { RT-PCR } & \text { mation }\end{array}$

\section{REFERENCES}

[1] Sellers JR. Myosin: a diverse superfamily. Biochem Biophys Acta 2000; 1496: 3-22.

[2] Goodson HV, Dawson SC. Multiplying myosins. Proc Natl Acad Sci USA 2006; 103: 3498-3499.

[3] Goodson HV, Spudich JA. Molecular evolution of the myosin family: Relationships derived from comparisons of amino acid sequences. Proc Natl Acad Sci USA 1993; 90: 659-663.

[4] Kieke MC, Titus MA, In: Schliwa M, Ed. The myosin superfamily: an overview. Molecular Motors, Wiley-VCH 2003; 3-44.

[5] Richards TA, Cavalier-Smith T. Myosin domain evolution and the primary divergence of eukaryotes. Nature 2005; 436: 1113-1118.

[6] Provance DW, Mercer JA. Myosin-V: head to tail. Cell Mol Life Sci 1999; 56: 233-242.

[7] Larson RE, Pitta DE, Ferro JA. A novel $190 \mathrm{kDa}$ calmodulinbinding protein associated with brain actomyosin. Braz J Med Biol Res 1998; 21: 213-217.

[8] Larson RE, Espindola FS, Espreafico EM. Calmodulin-binding proteins and calcium/calmodulin-regulated enzime ativities associated with brain actomyosin. J Neurochem 1990; 54: 1369-1383.

[9] Espindola FS, Espreafico EM, Coelho MV, et al. Biochemical and immunological characterization of p190-calmodulin complex from vertebrate brain: a novel calmodulin-binding myosin. J Cell Biol 1992; 118: 359-368.

[10] Mercer JA, Seperack PK, Strobel MC, Copeland NG, Jenkins NA Novel myosin-heavy chain encoded by murine dilute coat colour locus. Nature 1991; 349: 709-713.

[11] Johnston GC, Prendergast JA, Singer RA. The Saccharomyces cerevisiae MYO2 gene encodes an essencial myosin for vectorial transport of vesicles. J Cell Biol 1991; 113: 539-551. 
[12] Espreafico EM, Cheney RE, Matteoli M, et al. Primary structure and cellular localization of chicken brain myosin-V (p190), an unconventional myosin with calmodulin light chains. J Cell Biol 1992; 119: 1541-1557.

[13] Sanders G, Lichte B, Meyer HE, Kilimann MW. cDNA encoding the chicken ortholog of the mouse dilute gene product. Sequence comparison reveals a myosin I subfamily with conserved Cterminal domains FEBS Lett 1992; 311: 295-298.

[14] Cheney RE, Mooseker MS. Unconventional myosins. Curr Opin Cell Biol 1992; 4: 27-35.

[15] Zhao LP, Koslovsky JS, Reinhard J, et al. Cloning and characterization of myr6, an unconventional myosin of the dilute/myosin-V family. Proc Natl Acad Sci USA 1996; 93: 10826-10831.

[16] Rodriguez OC, Cheney RE. Human myosin-Vc is a novel class V myosin expressed in epithelial cells. J Cell Sci 2002; 115: 9911004.

[17] Welcomme RL. International introductions of inland aquatic species. FAO Fish Tech Pap 1988; 294: 1-318.

[18] Phillips RB, Nichols KM, DeKoning JJ, et al. Danzmann RG, Drew RG, Thorgaard GH. Assignment of Rainbow Trout Linkage Groups to Specific Chromosomes. Genetics 2006; 174: 1661-1670.

[19] Altschul SF, Gish W, Miller W, Myers EW, Lipman DJ. Basic local alignment search tool. J Mol Biol 1990; 215: 403-410.

[20] Thompson JD, Gibson TJ, Plewniak F, Jeanmougin F, Higgins DG. The CLUSTAL_X windows interface: flexible strategies for multiple sequence alignment aided by quality analysis tools. Nucleic Acids Res 1997; 25: 4876-4882.

[21] Kumar S, Tamura K, Nei M. MEGA 3: Integrated software for Molecular Evolutionary Genetics Analysis and sequence alignment. Brief Bioinformatics 2004; 5: 150-163.

[22] Seperack PK, Mercer JA, Strobel MC, Copeland NG, Jenkins NA. Retroviral sequences located within an intron of the dilute gene alter dilute expression in a tissue-specific manner. EMBO J 1995; 14 : 2326-2332.

[23] Huang J, MermallV, Strobel MC, et al. Molecular genetic dissection of mouse unconventional myosin-VA. Tail region mutations. Genetics 1998; 148: 1963-1972.

[24] Lambert J, Naeyaert JM, Callens T, De Paepe A, Messiaen L. Human myosin $\mathrm{V}$ gene produces different transcripts in a cell typespecific manner. Biochem Biophys Res Commun 1998; 252: 329333.

[25] Westbroek W, Lambert J, Bahadoran P, et al. Naeyaert J. Interactions of human myosin $\mathrm{Va}$ isoforms, endogenously expressed in human melanocytes, are tightly regulated by the tail domain. $\mathrm{J}$ Invest Dermatol 2003; 120: 465-475.

[26] Cope MJTV, Whisstock J, Rayment, Kendrick-Jones K. Conservation within the myosin motor domain: implications for structure and function. Structure 1996; 4: 969-987.

[27] Nelson DL, Cox MM. Lehninger Principles of Biochemistry. $4^{\text {th }}$. ed. Freeman and Company: New York 2005.

[28] Mooseker MS, Cheney RE. Unconventional myosins. Annu Rev Cell Dev Biol 1995; 11: 633-675.

[29] Wolenski JS. Regulation of calmodulin-binding myosins. Trends Cell Biol 1995; 5: 310-316.

[30] Larson RE. Myosin-V: a class of unconventional molecular motors. Braz J Med Biol Res 1996; 29: 309-318.

[31] Rhoads AR, Friedberg F. Sequence motifs for calmodulin recognition. FASEB J 1997; 11: 331-340.

[32] Tilelli CQ, Martins AR, Larson RE, Garcia-Cairasco N. Immunohistochemical localization of myosin $\mathrm{Va}$ in adult rat brain. J Neurosci 2003; 121: 573-586.

[33] Costa MCR, Mani F, Santoro W, Espreafico EM, Larson RE. Brain myosin-V, a calmodulin-carrying myosin, binds to calmodulindependent protein kinase II and activates its kinase activity. J Biol Chem 1999; 274: 15811-15819.

[34] Mani F, Espreafico EM, Larson RE. Myosin-V is present in synaptosomes from rat cerebral cortex. Braz J Med Biol Res 1994; 27 : 2639-2643.

[35] Prekeris R, Terrian DM. Brain myosin V is a synaptic vesicleassociated motor protein: evidence for a $\mathrm{Ca} 2+-d e p e n d e n t ~ i n t e r a c-$ tion with the synaptobrevin-synaptophysin complex. J Cell Biol 1997; 137: 1589-1601.

[36] Evans LL, Hammer J, Bridgman PC. Subcellular localization of myosin $\mathrm{V}$ in nerve growth cones and outgrowth from dilute-lethal neurons. J Cell Sci 1997; 110: 439-449.

[37] Tabb JS, Molyneaux BJ, Cohen DL, Kuznetsov SA, Langford GM. Transport of ER vesicles on actin filaments in neurons by myosin V. J Cell Sci 1998; 111: 3221-3234.

[38] Nascimento AA, Cheney RE, Tauhata SBF, Larson RE, Mooseker MS. Enzymatic characterization and functional domain mapping of brain myosin-V. J Biol Chem 1996; 271: 17561-17569.

[39] Pastural E, Barrat FJ, Dufourcq-Lagelouse R, et al. Griscelli disease maps to chromosome $15 \mathrm{q} 21$ and is associated with mutations in the myosin Va gene. Nat Genet 1997; 16: 289-292. 